\title{
Identification of Splicing Defects Caused by Mutations in the Dysferlin Gene
}

\author{
Virginie Kergourlay, ${ }^{1,2}$ Ghadi Raï, ${ }^{1,2}$ Gaëlle Blandin, ${ }^{1,2}$ David Salgado, ${ }^{1,2}$ Christophe Béroud, ${ }^{1,2,3}$ \\ Nicolas Lévy, ${ }^{1,2,3}$ Martin Krahn, ${ }^{1,2,3}$ and Marc Bartoli1,2,3* \\ ${ }^{1}$ Aix Marseille Université, GMGF, Marseille 13385, France; ${ }^{2}$ Inserm, UMR_S 910, Marseille 13385, France; ${ }^{3}$ Département de Génétique Médicale et \\ de Biologie Cellulaire, AP-HM, Hôpital d'Enfants de la Timone, Marseille 13385, France
}

\begin{abstract}
Missense, iso-semantic, and intronic mutations are challenging for interpretation, in particular for their impact in mRNA. Various tools such as the Human Splicing Finder (HSF) system could be used to predict the impact on splicing; however, no diagnosis result could rely on predictions alone, but requires functional testing. Here, we report an in vitro approach to study the impact of DYSF mutations on splicing. It was evaluated on a series of 45 DYSF mutations, both intronic and exonic. We confirmed splicing alterations for all intronic mutations localized in $5^{\prime}$ or $3^{\prime}$ splice sites. Then, we showed that DYSF missense mutations could also result in splicing defects: mutations c. $463 \mathrm{G}>\mathrm{A}$ and c.2641 $\mathrm{A}>\mathrm{C}$ abolished ESEs and led to exon skipping; mutations c.565C $>\mathrm{G}$ and c. $1555 \mathrm{G}>\mathrm{A}$ disrupted Exonic Splicing Enhancer (ESE), while concomitantly creating new $5^{\prime}$ or $3^{\prime}$ splice site leading to exonic out of frame deletions. We demonstrated that $20 \%$ of DYSF missense mutations have a strong impact on splicing. This minigene strategy is an efficient tool for the detection of splicing defects in dysferlinopathies, which could allow for a better comprehension of splicing defects due to mutations and could improve prediction tools evaluating splicing defects.

Hum Mutat 35:1532-1541, 2014. (C) 2014 Wiley Periodicals, Inc.
\end{abstract}

KEY WORDS: diagnostics tests; neuromuscular disease; splicing; dysferlin; DYSF

\section{Introduction}

Dysferlinopathies are disabling muscle diseases caused by mutations in DYSF (MIM \#603009, 2p13, GenBank NM_003494.3) [Bashir et al., 1998; Liu et al., 1998] the gene encoding the dysferlin protein implicated in muscle membrane repair [Bansal et al., 2003]. At the clinical level, dysferlinopathies present as different types of muscular dystrophies, including mainly LGMD2B (LGMD2B; MIM \#253601) and Miyoshi myopathy (MIM \#254130) [Bashir et al., 1998; Liu et al., 1998].

* Correspondence to: Marc Bartoli, Medical Genetics \& Functional Genomics, UMR S_910, INSERM-Aix-Marseille University, 27, Bd Jean Moulin, Marseille 13385, France. E-mail: marc.bartoli@univ-amu.fr
DYSF is a large-size gene ( $>230 \mathrm{kbp}, 55$ exons, 6243 coding base pairs) [Bashir et al., 1998; Liu et al., 1998; Aoki et al., 2001]. Mutational analysis of DYSF is confronted to a wide mutational spectrum, and associated interpretational difficulties for novel DYSF sequence variants, in particular putative splicing and missense variants. In a comprehensive analysis of the dysferlin mutational spectrum, using the locus-specific database UMD-DYSF (www.umd.be/DYSF/), we recently characterized the DYSF mutational spectrum based on all compiled DYSF disease-causing mutations reported in the literature [Blandin et al., 2012]. Among 266 different disease-causing mutations (from a total of 742 mutational entries corresponding to variations identified in 558 patients worldwide), 33.1\% corresponded to missense mutations and $17.3 \%$ to intronic mutations. In the last years, numerous bioinformatics tools have been developed to predict possible disease-causing effects of missense and/or intronic variants [Thusberg et al., 2011]. However, predicted pathogenic effects require functional validation to allow for definite conclusions. With regard to the diverse functional effects of missense changes at the protein level, diverse assays have been set up previously for dysferlin [Therrien et al., 2006; Fujita et al., 2007]. Noteworthy, in addition to functional consequences at the protein level, missense variants may be pathogenic due to deleterious effects on normal splicing of the messenger RNA in a proportion as high as 15\% [Cartegni et al., 2002]. Regarding intronic variants, the expected deleterious effects are also mainly related to abnormal splicing of the messenger RNA. Even though transcriptional analyses on patient samples may be used to evaluate abnormal splicing, the lack of availability of such material, which can be encountered in a diagnostic procedure, led to the development of splice-reporter assays [Gaildrat et al., 2010]. In the present work, we focused on adapting this functional assay to evaluate possible splicing defects caused by missense and intronic variants in the dysferlin gene, readily usable in particular or complex diagnostic applications.

\section{Materials and Methods}

\section{Mutations}

Mutations were chosen from the UMD-DYSF database (version 1.2; http://www.umd.be/DYSF/) [Blandin et al., 2012], excepted mutations c.1053+4A $>\mathrm{G}$ and c.3349-10G $>$ A for which we identified here for the first time in patients. No other mutation in DYSF for the patient with c. $1053+4 \mathrm{~A}>\mathrm{G}$ mutation was found; patient with c.3349-10G $>$ A mutation has the previously published mutation c.509C $>$ A. The mutation nomenclature follows the guidelines specified by http://www.hgvs.org/mutnomen/. 


\section{Predictions of the Pathogenicity of Mutations}

We used HSF (version 2.4.1) [Desmet et al., 2009] to predict the effect of mutations on splicing (http://www.umd.be/HSF/). We made all predictions with the DYSF transcript ENST00000258104.

We used the UMD-predictor algorithm [Frédéric et al., 2009], available through the online UMD-DYSF database [Blandin et al., 2012], to predict the deleterious effect of missense mutations.

\section{Cell Culture and Transfection}

HEK293T cells were cultured in DMEM-glutamax containing $15 \%$ of fetal bovine serum (Eurobio, Montpellier, France) and 1\% penicillin streptomycin amphotericin. We made transfections with promofectine (Promokine, Heidelberg, Germany) according to the manufacturer's instructions. We extracted RNA $48 \mathrm{hr}$ after the transfection. We made cotransfection of plasmid and AON using lipofectamine 2000 (Life Technologies, Carlsbad, CA).

\section{Splicing Minigene Reporter Assay}

We amplified exons and approximately 150 bp of introns surrounding each targeted exon from BAC RP11-1035E8 and RP111035B16 using the expand high-fidelity PCR system (Roche, Basel, Switzerland). Amplicons were subsequently cloned into the pCAS2 vector using BamHI and MluI sites (or BglII and AscI). PCR and digestions were performed using the NucleoSpin Gel and PCR clean Up (Macherey Nagel, Dürel, Germany), ligations using the T4 DNA ligase (Roche), and transformation using the library efficiency DH5 $\alpha$ competent cells (Life Technologies). Mutations were introduced into the construction using the Quick-change II XL site-directed mutagenesis kit (Agilent, Santa Clara, CA). We made transfections using Promofectin (Promokine) and $48 \mathrm{hr}$ after transfection we performed the transcriptional analysis.

\section{Design of the AON and Transfection}

We used AONs for characterization of Exonic Splicing Enhancer (ESE). All AONs were synthesized by Eurogentec (Seraing, Belgium) and contain 2'-O-methyl RNA and full-length phosphorothioate backbones. Sequences of these AONs are: AON targeting the sequence between c.555 and c.574 in exon 6 of DYSF containing the mutation c.565C>G: 5' GUGAAGGUACUUUCCUUGGG 3'; AON targeting the sequence between c.1547 and c.1566 in exon 18 of DYSF containing the mutation c.1555G>A: $5^{\prime}$ GUAGCAGGGCCUAAAAGUGG 3'; AON targeting the sequence between c.2622 and c.2636 in exon 25 of DYSF 5' GCAAAGACAGACAGC $3^{\prime}$. Mutations are underlined.

We made cotransfection of the p-CAS-construction with and without mutation and AON using Lipofectamine 2000 (Life Technologies) and $48 \mathrm{hr}$ after transfection we extracted RNAs.

\section{Transcriptional Study}

RNAs were extracted from cultured cells using the PureLink ${ }^{\mathrm{TM}}$ RNA Mini Kit (Life Technologies) and then treated using DNAse Free kit (Life Technologies). RNAs were reverse transcribed into cDNA using the high-capacity cDNA Reverse Transcription Kit (Life Technologies). PCR amplifications were performed with $2 \mu \mathrm{L}$ of RT products using the PCR master Mix (Promega, Madison, WI) using the primers forward 5' TGACGTCGCCGCCCATCAC $3^{\prime}$ and reverse $5^{\prime}$ ATTGGTTGTTGAGTTGGTTGTC $3^{\prime}$ located in exon A and exon B of pCAS2, respectively. PCR products were separated by electrophoresis in a $2 \%$ agarose gel stained with $5 \%$ of ethidium bromide. An additional purification step from the agarose gel was performed when several transcripts were present. We subcloned into pGEM $^{\circledR}$-T Easy Vector System I (Promega) when low quantities of PCR products were obtained. Finally, sequencing was performed using the Big DyeR Terminator V1-1 Cycle Sequencing Kit (Life Technologies).

\section{Results}

\section{The Splicing Reporter Minigene Assay Allows to Detect the Effect of Substitutions on Splicing}

The minigene strategy developed by Gaildrat et al. [2010] allows in vitro testing of deleterious effects of mutations on splicing. This test consists in integrating the exon of interest and the flanking intronic regions in constructs containing: the exon 2 (name here exon $\mathrm{A}$ ), the intron 2, and the exon 3 fused to the 122 bp of the exon 4 (name here exon $\mathrm{B}$ ) of the SERPING1/C1NH gene. The region of interest is integrated within intron 2 , and mutations to be analyzed are introduced using targeted mutagenesis. After transfecting the construct with and without the studied mutation into HEK cells, the splicing event is evaluated by RT-PCR and Sanger sequencing. We first confirmed the effectiveness of this assay in reproducing deleterious splicing events for a series of seven mutations (Table 1) for which the deleterious effect has been previously reported using transcriptional analyses on patient samples. Representative examples are illustrated in Figure 1A and D for mutation c. $2643+2 T>G$, which disrupts the natural $5^{\prime}$ splice site of exon 25 resulting in the exon 25 skipping and c. $1353+1 \mathrm{G}>\mathrm{A}$, which disrupts the natural $5^{\prime}$ splice site of intron 14 resulting in the inclusion of 178 bp of intron 14 , respectively. To determine the specificity of our test, we evaluated two mutations for which a deleterious effect at the protein level was reported, whereas a deleterious effect on splicing was excluded for one of them. Indeed, mutation c.4022T $>C$ (p.Leu1341Pro) leads to an accumulation in the endoplasmic reticulum within many patient's muscle fibers [Wenzel et al., 2006]. This accumulation suggests a dysferlin aggregation leading to the degradation by the autophagy/lysosome endoplasmic reticulum-associated degradation system [Fujita et al., 2007]. No sequence abnormalities due to this mutation were detected by RNA analysis [Cacciottolo et al., 2011]. Moreover, it has been shown that mutation c.1663C $>\mathrm{T}$ (p.Arg555Trp) leads to protein degradation by the proteasome [Azakir et al., 2012a]. Accordingly, we detected no splicing abnormalities due to these mutations using the minigene strategy, suggesting that no false-positive results were obtained with this approach (Table 1).

\section{Abolition of Natural Splice Sites}

The minigene strategy was used to evaluate mutations localized in natural splice sites for which the effect on splicing has not been previously investigated at the transcriptional level due to limited availability of patient samples (Table 2 ). Regarding seven intronic mutations localized in $5^{\prime}$ splice sites (positions +1 to +5 ), all tested mutations led to exon skipping (Fig. 1A). No effect on splicing was observed for the mutation $c .5767+6 \mathrm{C}>\mathrm{T}$ in agreement with bioinformatics predictions provided by HSF. Only one missense mutation, c.5525G $>\mathrm{A}$ (p.Gly1842Asp), is localized in a $5^{\prime}$ splice site. It targets the last nucleotide of exon 49 (Table 3 ). This mutation disrupts this site leading to the in-frame skipping of exon 49. 


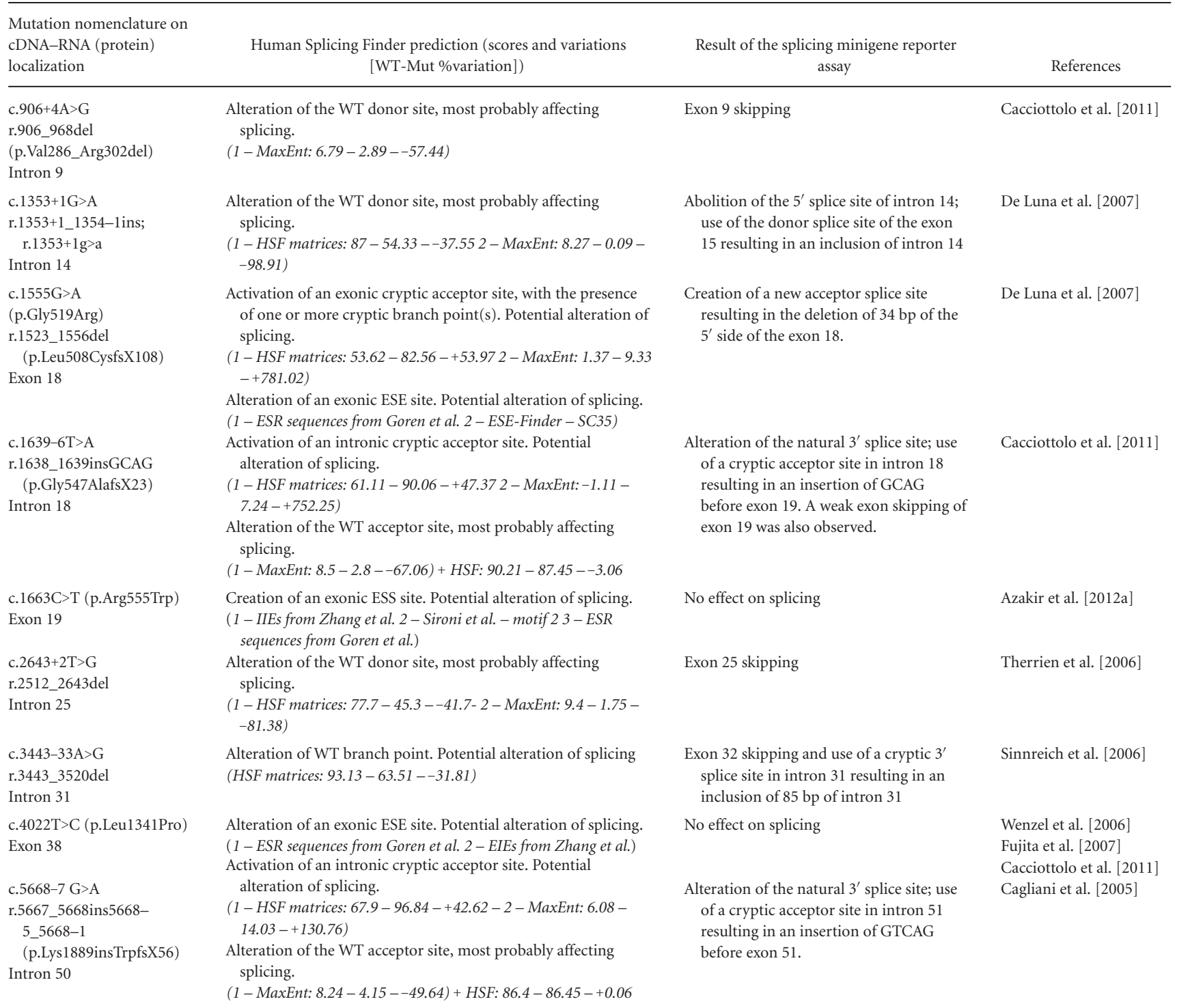

The scores of pathogenicity of mutation c.1555G >A (p.Gly519Arg), c.1663C > T (p.Arg555Trp), and c.4022T >C (p.Leu1341Pro) given by UMD-DYSF predictor are 100, 88, and 71, respectively.

The five intronic mutations localized in a $3^{\prime}$ splice site (positions -1 and -2 ) led to abnormal splicing. Mutation c.1354-1G>A located in intron 14 led to exon 15 skipping. The four other mutations resulted in the activation of a cryptic $3^{\prime}$ splice site, resulting in an insertion or a deletion at the mRNA level. For mutation c.938-1G $>A$, we identified two transcripts. This mutation disrupted the natural $3^{\prime}$ splice site while simultaneously activating a cryptic $3^{\prime}$ splice site. It resulted into a shorter out-of-frame mRNA (deletion of the first nucleotide [G] of exon 11). A cryptic $3^{\prime}$ splice site located in intron 10 was also used, resulting in the inclusion of $135 \mathrm{bp}$ of intron 10. Mutation c.1285-2A $>\mathrm{G}$ disrupted the natural splice site and led to the activation of a cryptic $3^{\prime}$ splice site located in exon 14 . It resulted into a shorter out-of-frame mRNA (deletion of the eight first nucleotides of exon 14). Mutation c.3349-2A $>\mathrm{G}$ disrupted the natural splice site and led to the use of two cryptic $3^{\prime}$ splice sites in intron 30 and exon 31 . It resulted in the insertion of $60 \mathrm{bp}$ of intron 30 and the deletion of the 21 first nucleotides of exon 31 , respectively (Fig. 1B). Similarly, mutation c.3703-1G $>$ A disrupted the natural $3^{\prime}$ splice site while simultaneously activating a cryptic $3^{\prime}$ splice site. This resulted in the deletion of the first nucleotide $(\mathrm{G})$ of exon 34. A weak skipping of exon 34 was also observed. The missense mutation c.5201A $>$ G (p.Glu1734Gly), which is located in the first nucleotide of the exon 47, did not impact the splicing in agreement with bioinformatics predictions provided by HSF (Table 4).

Two mutations located in the polypyrimidine tract region, c. 1639-6T $>A$ and c.5668-7 G>A, led to the use of cryptic $3^{\prime}$ splice sites located in intron 18 and 50, which resulted in the insertion of GCAG and GTCAG, respectively. No perturbation of splicing is detected for the intronic mutation c.3349-10G $>A$, in accordance with the HSF prediction (Table 1).

Mutation c.3443-33A $>\mathrm{G}$ disrupted the branch point, leading to the production of two transcripts. The first transcript corresponds to the skipping of exon 32, and the second to the inclusion of $85 \mathrm{bp}$ of intron 31 (Fig. 1C). The exon 32 skipping transcript was previously described by Sinnreich et al. [2006], whereas the second transcript was not reported. In agreement with our in vitro assay results, HSF 


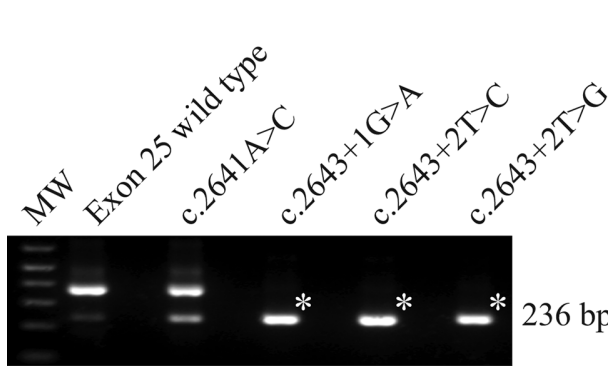

A

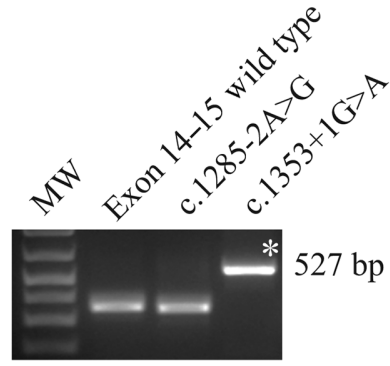

$\mathrm{D}$

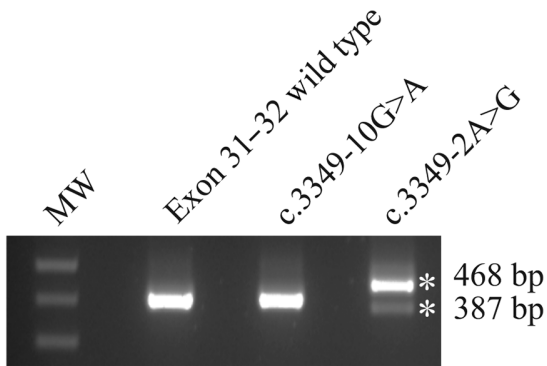

B

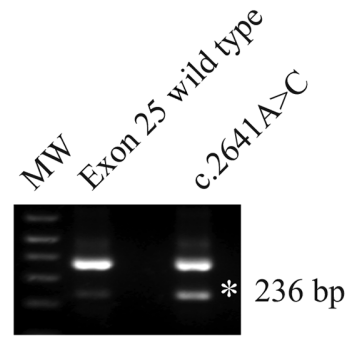

E

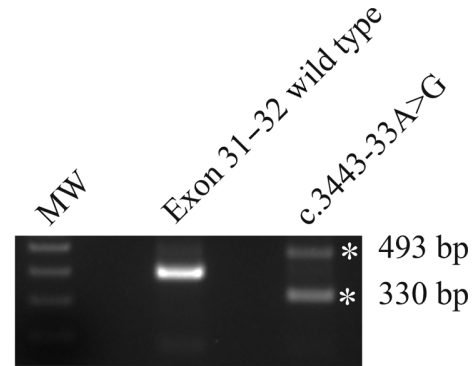

$\mathrm{C}$

Figure 1. Transcriptional analysis of DYSFmutations. We used the splicing reporter minigene assay to detect the impact on splicing of mutations. In each case, transfections into HEK 293 cells were performed both using a WT construction and the mutant pCAS2 construction. Forty-eight hours after transfection, transcriptional analysis was performed. Representative examples of splicing effects are shown. All effects on splicing identified using RT-PCR were further confirmed using Sanger sequencing. Abnormal transcripts are indicated with a white asterisk. A: Exon skipping due to mutations c. $2643+1 \mathrm{G}>\mathrm{A} ; \mathrm{c} .2643+2 \mathrm{~T}>\mathrm{C} ; \mathrm{c} .2643+2 \mathrm{~T}>\mathrm{G}$ located in 5 'splice sites. B: Use of two cryptic $3^{\prime}$ splice sites in intron 30 and exon 31 due to mutation c.3349-2A>G, resulting in the insertion of $60 \mathrm{bp}$ of intron 30 and the deletion of the 21 first nucleotides of exon 31 , respectively. C: Disruption of the branch point due to mutation c.3443-33A $>\mathrm{G}$, leading to the production of two transcripts. The 330-bp transcript corresponds to the skipping of exon 32, and the 493-bp transcript to the inclusion of $85 \mathrm{bp}$ of intron 31. D: Inclusion of intron 14 due to the mutation c.1353+1G>A affecting the $5^{\prime}$ splice site. E: Partial exon skipping due to missense mutation c.2641A $>C$. F: The creation of a new $3^{\prime}$ splice site due to mutation c. $1555 \mathrm{G}>\mathrm{A}$, resulting in a deletion of $34 \mathrm{bp}$ in the $5^{\prime}$ extremity of exon 18.

predicted the presence of a cryptic $3^{\prime}$ splice site, which has a strong consensus value $(\mathrm{CV})$ of 85.1. Moreover, a cryptic branch point with a CV of 96.1 is located 31 bp upstream of the intron/exon junction of this cryptic splice site (Table 1).

In conclusion, we showed that this in vitro DYSF minigene assay enabled the detection of splicing abnormalities due to mutations abolishing natural splice sites. Indeed, because the probability that mutations located within these signals impair the splicing mechanism is very high, the use of such assay is highly recommended.

\section{Analysis of Possible Splicing Defects Caused by Exonic Substitutions}

The minigene assay was used to test the impact on splicing of exonic substitutions outside of $3^{\prime}$ and $5^{\prime}$ splice sites. Possible deleterious effects of such mutations are more difficult to predict using bioinformatics strategies. We tested 25 exonic substitutions (missense mutations). We showed that five missense mutations led to abnormal splicing, corresponding to $20 \%$ of tested exonic substitutions (Tables 1 and 3). For mutation c.1555G $>A$ (p.Gly519Arg), the observed deleterious effect on splicing was previously reported and confirmed using the splicing assay, with accurate correlation. We also showed deleterious effects on splicing for the mutations c.463G $>$ A (p.Gly155Arg); c.2641A $>C$ (p.Thr881Pro); c.565C $>$ G (p.Leu189Val); and c.5525G $>$ A (p.Gly1842Asp) on the splicing mechanism, which had never been reported. Mutation c.5525G $>$ A disrupted the $5^{\prime}$ splice site as described in the second paragraph. Mutations c. $463 \mathrm{G}>\mathrm{A}$ and c. $2641 \mathrm{~A}>\mathrm{C}$ resulted in a partial skipping of the corresponding exon. Both c.565C $>\mathrm{G}$ and c.1555G $>\mathrm{A}$ mutations activated a cryptic splice site.

\section{Exonic Substitutions Could Impact ESE/ESS Signals}

Exonic substitutions may alter the splicing by modifying ESE and ESS. Mutations c. $463 \mathrm{G}>\mathrm{A}$ and c.2641A $>\mathrm{C}$ (Fig. 1E) resulted in a partial skipping of exon 6 and 25, respectively. Skipping of exon 6 is predicted to create a frameshift leading to a premature stop codon. Skipping of exon 25 induced the in-frame deletion of this exon. To confirm whether mutation c.2641A $>C$ disrupted an ESE, we designed an AON targeting the exon 25 region between c. 2622 and c.2636. Cotransfection of the AON and the pCAS2-DYSF exon 25 with and without the mutation induced the skipping of exon 25 (Fig. 2C). This skipping was stronger in the mutant because the mutation itself led to exon 25 skipping. In this situation, we thus observed a combination of the effect of the AON and the mutation. These results indicate that the targeted region (c.2622-c.2636) is necessary for a correct splicing and most probably contains an ESE.

\section{Exonic Substitutions Could Impact Splicing by Creating Cryptic Splice Sites}

Exonic substitutions may also create new splice sites and concomitantly alter auxiliary splicing signals. Abnormal transcripts were observed for mutations c. $565 \mathrm{C}>\mathrm{G}$ and c. $1555 \mathrm{G}>\mathrm{A}$. Mutation c. $565 \mathrm{C}>\mathrm{G}$, localized in exon 6 of $D Y S F$, led to the production of the 
Table 2. Analysis of Splicing Effects of Intronic Mutations Using the Minigene Assay

Mutation nomenclature

on cDNA localization Human Splicing Finder prediction (scores and variations [WT-Mut \% variation])

Result of the splicing minigene reporter assay

\begin{tabular}{|c|c|c|}
\hline $\begin{array}{l}\text { c. } 663+1 \mathrm{G}>\mathrm{C} \\
\text { Intron } 6\end{array}$ & $\begin{array}{l}\text { Alteration of the WT donor site, most probably affecting splicing. } \\
(1-\text { HSF matrices: } 83.82-58.81--29.842-\text { MaxEnt: } 5.99--2,27--37.9)\end{array}$ & Exon 6 skipping \\
\hline c. $2643+1 \mathrm{G}>\mathrm{A}$ & Alteration of the WT donor site, most probably affecting splicing. & Exon 25 skipping \\
\hline Intron 25 & ( $1-$ HSF matrices: $77.7-45.04--42,03-2-$ MaxEnt: $9.4-1.22--8702)$ & \\
\hline c. $3843+1 \mathrm{G}>\mathrm{A}$ & Alteration of the WT donor site, most probably affecting splicing. & Exon 34 skipping \\
\hline Intron 34 & (1-HSF matrices: $93.18-60.51--35.06-2-$ MaxEnt: $9.16-0.98--89.3)$ & \\
\hline c. $2643+2 \mathrm{~T}>\mathrm{C}$ & Alteration of the WT donor site, most probably affecting splicing. & Exon 25 skipping \\
\hline Intron 25 & ( $1-$ HSF matrices: $77.7-45.56--41.36-2-$ MaxEnt: $9.4-1.65--82.45)$ & \\
\hline c. $3843+2 \mathrm{~T}>\mathrm{A}$ & Alteration of the WT donor site, most probably affecting splicing. & Exon 34 skipping \\
\hline Intron 34 & (1-HSF matrices: $93.18-60.77--34.78-2-$ MaxEnt: $9.16-0.97--89.41)$ & \\
\hline $\begin{array}{l}\text { c. } 1053+4 \mathrm{~A}>\mathrm{G} \\
\text { Intron } 11\end{array}$ & $\begin{array}{l}\text { Alteration of the WT donor site, most probably affecting splicing. } \\
(1-\text { HSF matrices: } 79.1-61.61--22.112-\text { MaxEnt: } 7.21-1.37--81) \\
\text { Activation of an intronic cryptic donor site. Potential alteration of splicing. } \\
(1-\text { HSF matrices: } 61.62-72.19-+17.15)\end{array}$ & Exon 11 skipping \\
\hline $\begin{array}{l}\text { c. } 1053+5 \mathrm{G}>\mathrm{A} \\
\text { Intron } 11\end{array}$ & $\begin{array}{l}\text { Alteration of the WT donor site, most probably affecting splicing. } \\
\text { (1-HSF matrices: } 79.1-46.08--41.742-\text { MaxEnt: } 7.21--2.93--140.64)\end{array}$ & Exon 11 skipping \\
\hline c. $5767+6 \mathrm{C}>\mathrm{T}$ & No significant splicing motif alteration detected. & No effect on splicing \\
\hline Intron 51 & This mutation has probably no impact on splicing. & \\
\hline $\begin{array}{l}\text { c. } 938-1 G>A \\
\text { Intron } 10\end{array}$ & $\begin{array}{l}\text { Alteration of the WT acceptor site, most probably affecting splicing. } \\
(1-\text { HSF matrices: } 84.06-55.11--34.442-\text { MaxEnt: } 8.16--0.58--107.11) \\
\text { Activation of an intronic cryptic acceptor site. Potential alteration of splicing. } \\
(1-\text { HSF matrices: } 55.87-84.82-+51.822-\text { MaxEnt: }-2.31-5.63-+343.72)\end{array}$ & $\begin{array}{l}\text { Abolition of the } 3^{\prime} \text { splice site of the exon } 11 \text {. Use of a cryptic } \\
\text { acceptor splice site resulting in the inclusion of } 135 \text { bp of } \\
\text { the intron } 10 \text {. The mutation creates simultaneously a new } \\
3^{\prime} \text { splice site, resulting in the deletion of the first G of the } \\
\text { exon } 11 .\end{array}$ \\
\hline $\begin{array}{l}\text { c. } 1354-1 \mathrm{G}>\mathrm{A} \\
\text { Intron } 14\end{array}$ & $\begin{array}{l}\text { Alteration of the WT acceptor site, most probably affecting splicing. } \\
(1-\text { HSF matrices: } 91.23-62.28--31.732-\text { MaxEnt: } 8.1--0,64--107.9)\end{array}$ & Exon 15 skipping \\
\hline $\begin{array}{l}\text { c. } 3703-1 \mathrm{G}>\mathrm{A} \\
\text { Intron } 33\end{array}$ & $\begin{array}{l}\text { Alteration of the WT acceptor site, most probably affecting splicing. } \\
\text { ( } 1-\text { HSF matrices: } 79.38-50.44--36.46-2-\text { MaxEnt: } 6.48--2-26--134.88) \\
\text { Activation of an intronic cryptic acceptor site. Potential alteration of splicing. } \\
\text { (1-HSF matrices: } 49.29-78.24-+58.73-2-\text { MaxEnt: }-3.45-4.5-+230.43)\end{array}$ & $\begin{array}{l}\text { Abolition of the } 3^{\prime} \text { splice site of intron } 33 \text {, use of a cryptic } 3^{\prime} \\
\text { splice site resulting in the deletion of a } \mathrm{G} \text { at the beginning } \\
\text { of the exon } 34 \text {. A weak exon } 34 \text { skipping is also present. }\end{array}$ \\
\hline c. $1285-2 A>G$ & Alteration of the WT acceptor site, most probably affecting splicing. & Abolition of the natural $3^{\prime}$ splice site, use of a cryptic \\
\hline Intron 13 & (1-HSF matrices: $80.14-51.19--36.122-$ MaxEnt: $10.28-2.32--77.43)$ & $\begin{array}{l}\text { acceptor splice site resulting in the deletion of } 8 \text { bp in } 5^{\prime} \\
\text { side of exon } 14 \text {. }\end{array}$ \\
\hline c. $3349-2 A>G$ & Alteration of the WT acceptor site, most probably affecting splicing. & Abolition of the natural $3^{\prime}$ splice site; use of two cryptic \\
\hline Intron 30 & (1-HSF matrices: $91.51-62.57--31.62-2-$ MaxEnt: $6.45--1.49--123.1)$ & $\begin{array}{l}\text { acceptor splice sites resulting in the insertion of } 60 \mathrm{bp} \text { of } \\
\text { the intron } 30 \text { and the deletion of } 21 \mathrm{bp} \text { of the } 5^{\prime} \text { part of } \\
\text { exon } 31 .\end{array}$ \\
\hline c. $3349-10 \mathrm{G}>\mathrm{A}$ & No significant splicing motif alteration detected. & No effect on splicing. \\
\hline Intron 30 & This mutation has probably no impact on splicing. & \\
\hline
\end{tabular}

normal transcript and an additional transcript presenting an out-offrame deletion of the last $103 \mathrm{bp}$ of this exon through the activation of a cryptic $5^{\prime}$ splice site. The CV of the wild-type (WT) cryptic site evaluated using HSF is 60.2, and for the mutant it increased to 72.2. The natural $5^{\prime}$ splice site has a strength of 84.6. The strengths' difference between the natural and the cryptic sites are not in favor of the activation of the cryptic site. We therefore hypothesized that the mutation may also impair a natural ESE required for the recognition of the natural site. To verify this hypothesis, we cotransfected an AON targeting the region between C.555 and c.574, which includes the mutation and the cryptic $5^{\prime}$ splice site, and the pCAS2-DYSF exon 6 with and without the mutation (Fig. 2A). We showed that the AON induced the exon 6 skipping, more strongly in the mutant because no mismatch was present in this case. This result suggested that the mutation c.565C $>\mathrm{G}$ also inactivated an auxiliary splicing signal, most probably an ESE.

Mutation c. $1555 \mathrm{G}>\mathrm{A}$ in exon 18 of DYSF created a new $3^{\prime}$ splice site used instead of the natural $3^{\prime}$ splice site. It resulted in an outof-frame deletion of $34 \mathrm{bp}$ in the $5^{\prime}$ extremity of exon 18 (Fig. $1 \mathrm{~F}$ ). The CV of the natural cryptic site is 53.6 and 82.6 for the mutant. The natural $3^{\prime}$ splice site has a CV of 86.2. As for $3^{\prime}$ splice sites, the site selection is strongly dependent of the branch point; a cryptic $3^{\prime}$ splice site localized downstream of the natural site is rarely activated. We therefore hypothesized that the mutation may simultaneously impair an auxiliary splicing signal required for the natural splice site recognition. Cotransfection of an $\mathrm{AON}$ targeting the mutant region between c.1547 and c.1566, and the pCAS2-DYSF exon 18 with and without the mutation, induced the skipping of the exon 18 in the natural sequence (Fig. 2B). In the mutant, only a partial skipping was observed. This result suggested that the mutation also inactivated an auxiliary splicing signal, most probably an ESE. Furthermore, HSF predicts the creation of an ESS (new hnRNAP1-binding motif) that inhibits the proper recognition of the natural $3^{\prime}$ splice site.

These two missense mutations could therefore both create new splice site signals and concomitantly reduce the natural $5^{\prime}$ or $3^{\prime}$ splice sites recognition by disrupting an ESE or creating an ESS, showing that in these cases a combined effect was necessary for the deleterious effect on splicing.

\section{Discussion}

A major problem for the detection of splicing abnormalities in diagnosis is the limited access to patient muscle biopsy material for transcriptional studies. To overcome this problem, transcriptional analyses have been developed on patient monocytes [De Luna et al., 2007] allowing for detection of splicing effects, but requiring specific sampling and cell sorting methods. As another alternative, we applied a previously developed splicing reporter minigene assay [Gaildrat et al., 2010] for the DYSF gene in order to detect DYSF splicing defects due to substitutions, and evaluated this assay with 45 mutations. The development of this splicing assay widens the possibility 
Table 3. Identification of Splicing Effects of Missense Substitutions Using the Minigene Assay

\begin{tabular}{|c|c|c|c|}
\hline $\begin{array}{l}\text { Mutation nomenclature } \\
\text { on cDNA } \\
\text { (protein)_localization }\end{array}$ & $\begin{array}{l}\text { UMD-DYSF } \\
\text { predictor } \\
\text { prediction }\end{array}$ & Human Splicing Finder prediction (scores and variations [WT-Mut \%variation]) & $\begin{array}{l}\text { Result of the splicing } \\
\text { minigene reporter assay }\end{array}$ \\
\hline $\begin{array}{l}\text { c. } 463 \mathrm{G}>\mathrm{A} \\
\text { (p.Gly155Arg) } \\
\text { Exon } 6\end{array}$ & 82 & $\begin{array}{l}\text { Activation of an exonic cryptic acceptor site. Potential alteration of splicing. } \\
(1-H S F \text { matrices: } 44.79-73.74-+64.63) \\
\text { Creation of an exonic ESS site. Potential alteration of splicing. } \\
(1-\text { ESR Sequences from Goren et al. } 2-\text { HSF matrices - hnRNP A1) } \\
\text { Alteration of an exonic ESE site. Potential alteration of splicing. } \\
(1-P E S E \text { octamers from Zhang and Chasin } 2-E S E-F i n d e r-S F 2 / A S F(I g))\end{array}$ & Partial skipping of the exon 6 \\
\hline $\begin{array}{l}\text { c. } 565 \mathrm{C}>\mathrm{G} \\
(\mathrm{p} . \text { Leu } 189 \mathrm{Val}) \\
\text { Exon } 6\end{array}$ & 29 & $\begin{array}{l}\text { Activation of an exonic cryptic donor site. Potential alteration of splicing. } \\
(1-H S F \text { matrices: } 60.2-72.21-+19.95) \\
\text { Activation of an exonic cryptic donor site. Potential alteration of splicing. } \\
(1-H S F \text { matrices: } 40.82-67.65-+67.65) \\
\text { Activation of an exonic cryptic acceptor site, with the presence of one or more cryptic branch } \\
\text { point(s). Potential alteration of splicing. } \\
(1-H S F \text { matrices: } 38.76-67.71-+74.69) \\
\text { Creation of an exonic ESS site. Potential alteration of splicing. } \\
(1-\text { PESS octamers from Zhang and Chasin } 2-\text { ESR sequences from Goren et al. }) \\
\text { Alteration of an exonic ESE site. Potential alteration of splicing. } \\
\text { (1- HSF matrices }-9 G 82-\text { RESCUE ESE hexamers } 3-\text { EIEs from Zhang et al. } 4-\text { ESR sequences } \\
\text { from Goren et al. })\end{array}$ & $\begin{array}{l}\text { Creation of a } 5^{\prime} \text { splice site, } \\
\text { resulting in the deletion of } \\
\text { the } 103 \text { bp of } 3^{\prime} \text { end of the } \\
\text { exon } 6\end{array}$ \\
\hline $\begin{array}{l}\text { c. } 2641 \mathrm{~A}>\mathrm{C} \\
\text { (p.Thr881Pro) } \\
\text { Exon } 25\end{array}$ & 76 & $\begin{array}{l}\text { Alteration of an exonic ESE site. Potential alteration of splicing. } \\
(1-\text { RESCUE ESE hexamers - } 2-\text { ESE-Finder - SRp } 40-3-\text { HSF matrices - } 9 G 8-4-\text { EIEs from } \\
\text { Zhang et al.) }\end{array}$ & Exon 25 skipping \\
\hline $\begin{array}{l}\text { c. } 5525 \mathrm{G}>\mathrm{A} \\
\text { (p.Gly1842Asp) } \\
\text { Exon } 49\end{array}$ & 100 & $\begin{array}{l}\text { Alteration of the WT donor site, most probably affecting splicing. } \\
(1-\text { HSF matrices: } 81.08-61.12--24.62-2-\text { MaxEnt: } 5.76--0.13--102.26)\end{array}$ & Exon 49 skipping \\
\hline
\end{tabular}

for the analysis of DYSF splicing defects, by offering the possibility of transcriptional analysis either on muscle tissue or monocytes, or using this novel in vitro assay depending on the sampling and laboratory context.

We showed that mutations in $5^{\prime}$ splice sites resulted in exon skipping in nine out of 11 mutations. For instance, the mutation c. $1353+1 \mathrm{G}>\mathrm{A}$ that inactivates the $5^{\prime}$ splice site of intron 14 induced the inclusion of the entire intron 14 (178 bp). Mutations in $3^{\prime}$ splice sites located in -1 and -2 (five mutations) and in the polypyrimidine tract region in position -6 and -7 (two mutations), mostly led to the activation of cryptic $3^{\prime}$ sites (six out of seven). Only mutation c.1354-1G>A located in intron 14 led to an exon skipping.

The different observed effects for mutations involving $5^{\prime}$ and $3^{\prime}$ splice sites were probably due to the sequential processing of pre-mRNA. Indeed, $5^{\prime}$ splice sites are involved early in the splicing mechanism as association of U1 snRNP with the $5^{\prime}$ splice site is the first step for the formation of the E complex. Mutations located in this site may thus prevent the initiation of the splicing mechanism, resulting mostly in the exon exclusion. Contrarily, it has been previously shown that $3^{\prime}$ splice sites are dispensable for spliceosome assembly, and are determined by a scanning process that recognizes the first AG located $3^{\prime}$ of the branch point/polypyrimidine tract, irrespective of the distance or sequence environment [Smith et al., 1989]. When $3^{\prime}$ splice sites are disrupted, we assumed that the splicing mechanism has already been initiated and the spliceosome interacts with a downstream $3^{\prime}$ cryptic site to complete the splicing. Furthermore, the force of the $3^{\prime}$ splice site created by mutation c. $2456 \mathrm{G}>\mathrm{A}$ is 94.44 , which is higher than the force of the natural splice site (86.62). We consider that the natural $3^{\prime}$ splice site is detected before and is therefore used despite the stronger strength of the site created by mutation located downstream.

We showed that the c.3443-33A $>$ G mutation disrupted the branch point of intron 31 . The exon 32 skipping due to this mutation was previously described by Sinnreich et al. [2006]. The authors reported a mildly affected patient, compound heterozygous for this mutation and the out-of-frame mutation c.4872_4876delinsCCCC. We showed that mutation c.3443-33A $>$ G led to the production of a second transcript corresponding to the inclusion of $85 \mathrm{bp}$ of intron 31 resulting from the use of another branch point $/ 3^{\prime}$ splice site. Sinnreich et al. [2006] did not report the presence of this second transcript; however, they reported that only $10 \%$ of dysferlin is expressed at the protein level in the patient. In parallel, another study has shown that dysferlin lacking exon 32 is stable [Azakir et al., 2012b]. We showed that the c.3443-33A>G mutation led to the expression of two transcripts, so we hypothesized that the exon 32 skipping represents a fraction inferior to $50 \%$ of the produced transcripts.

Previous analysis on patient's RNA showed that different mutations of DYSF disrupt splicing [Cagliani et al., 2005; Therrien et al., 2006; De Luna et al., 2007; Cacciottolo et al., 2011]. We confirmed here the deleterious effects on splicing for all of these previously described mutations. Moreover, no splicing defect was observed for two mutations with a reported impact on the dysferlin protein. Altogether, these results indicate that the minigene strategy is able to discriminate splicing defects.

Noteworthy, two differences were seen: the presence of a second weak transcript corresponding to an exon 19 skipping detected for the mutation c.1639-6T $>A$, and the inclusion of $85 \mathrm{bp}$ of intron 31 for the mutation c.3443-33A $>\mathrm{G}$ described in the previous paragraph.

However, in the cases for which the deleterious effect was not reported, we detected both normal and abnormal transcripts in experiments with and without mutations. We concluded that corresponding mutations had a strong enough impact on splicing to be detected by our in vitro assay, but we were not able to predict the percentage of the abnormal transcripts in patients with this test. Indeed, the genomic context and the different cellular type of this minigene strategy may not be perfectly adequate for quantification. When possible, we had constructed a minigene with two exons of $D Y S F$ to preserve the nearby genomic context within the DYSFlocus. 
Table 4. Missense Substitutions with no Deleterious Effect of Splicing Identified Using the Minigene Assay

\begin{tabular}{|c|c|c|c|}
\hline $\begin{array}{l}\text { Mutation nomenclature on } \\
\text { cDNA (protein) localization }\end{array}$ & $\begin{array}{l}\text { UMD-DYSF } \\
\text { predictor } \\
\text { prediction }\end{array}$ & Human Splicing Finder prediction (scores and variations [WT-Mut \%variation]) & $\begin{array}{l}\text { Result of the splicing } \\
\text { minigene reporter } \\
\text { assay }\end{array}$ \\
\hline $\begin{array}{l}\text { c.509C>A (p.Ala170Glu) } \\
\text { Exon } 6\end{array}$ & 71 & $\begin{array}{l}\text { Activation of an exonic cryptic acceptor site, with the presence of one or more cryptic branch } \\
\text { point(s). Potential alteration of splicing. } \\
\text { (HSF matrices: } 36.18-65.13-+80.02 \text { ) } \\
\text { Creation of an exonic ESS site. Potential alteration of splicing. } \\
\text { (1-PESS octamers from Zhang and Chasin - } 2 \text { - Sironi et al. - Motif 1-3-ESR sequences from } \\
\text { Goren et al. } 4 \text { - HSF matrices - hnRNP A1) }\end{array}$ & No effect on splicing \\
\hline $\begin{array}{l}\text { c.536G>A (p.Gly179Asp) } \\
\text { Exon } 6\end{array}$ & 80 & $\begin{array}{l}\text { Alteration of an exonic ESE site. Potential alteration of splicing. } \\
\text { (ESE-Finder-HSF matrices) }\end{array}$ & No effect on splicing \\
\hline $\begin{array}{l}\text { c. } 851 \mathrm{~T}>\mathrm{C}(\mathrm{p} . \mathrm{Ile} 284 \mathrm{Thr}) \\
\text { Exon } 8\end{array}$ & 93 & $\begin{array}{l}\text { Creation of an exonic ESS site. Potential alteration of splicing. } \\
\text { (1-Sironi et al. - Motif } 32 \text { - Fas-ESS hexamers) }\end{array}$ & No effect on splicing \\
\hline $\begin{array}{l}\text { c. } 1016 \mathrm{C}>\mathrm{G}(\mathrm{p} . \text { Thr339Arg }) \\
\text { Exon } 11\end{array}$ & 81 & $\begin{array}{l}\text { Activation of an exonic cryptic acceptor site, with the presence of one or more cryptic branch } \\
\text { point(s). Potential alteration of splicing. } \\
(1-\text { HSF matrices: HSF: } 44.12-73.06-+65.59) \\
\text { Creation of an exonic ESS site. Potential alteration of splicing. } \\
\text { (1-PESS octamers from Zhang and Chasin } 2 \text { - Sironi et al. - motif } 13-\text { ESR sequences from } \\
\text { Goren et al.) }\end{array}$ & No effect on splicing \\
\hline $\begin{array}{l}\text { c. } 1120 \mathrm{G}>\mathrm{C}(\mathrm{p} . \text { Val374Leu }) \\
\text { Exon } 12\end{array}$ & 29 & $\begin{array}{l}\text { Alteration of an exonic ESE site. Potential alteration of splicing. } \\
(1-E S E-F i n d e r-S F 2 / A S F 2-E S E-F i n d e r-S F 2 / A S F(I g) 3-E S E-F i n d e r-S R p 554-H S F \\
\text { matrices-9G8) }\end{array}$ & No effect on splicing \\
\hline $\begin{array}{l}\text { c.1165G }>C \text { (p.Glu389Gln) } \\
\text { Exon } 12\end{array}$ & 47 & $\begin{array}{l}\text { Creation of an exonic ESS site. Potential alteration of splicing. } \\
\text { (1-Sironi et al. - motif } 32-\text { ESR Sequences from Goren et al.) } \\
\text { Alteration of an exonic ESE site. Potential alteration of splicing. } \\
\text { (1-ESE-Finder - SF2/ASF } 2 \text { - ESE-Finder - SF2/ASF(Ig) 3-EIEs from Zhang et al. } 4 \text { - HSF } \\
\text { Matrices - 9G8) }\end{array}$ & No effect on splicing \\
\hline $\begin{array}{l}\text { c.1168G>A (p.Asp390Asn) } \\
\text { Exon } 12\end{array}$ & 41 & $\begin{array}{l}\text { Alteration of an exonic ESE site. Potential alteration of splicing. } \\
\text { (1-ESE-Finder-SF2/ASF } 2-\text { EIEs from Zhang et al.) }\end{array}$ & No effect on splicing \\
\hline $\begin{array}{l}\text { c. } 1369 \mathrm{G}>\mathrm{A} \text { (p.Glu457Lys) } \\
\text { Exon } 15\end{array}$ & 59 & $\begin{array}{l}\text { Creation of an exonic ESS site. Potential alteration of splicing. } \\
\text { (1-ESR sequences from Goren et al. } 2 \text { - PESS octamers from Zhang and Chasin) } \\
\text { Alteration of an exonic ESE site. Potential alteration of splicing. } \\
\text { (1-EIEs from Zhang et al. } 2 \text { - ESE-Finder - SRp55 } 3 \text { - RESCUE ESE hexamers } 4 \text { - HSF Matrices } \\
\text { - 9G8) }\end{array}$ & No effect on splicing \\
\hline $\begin{array}{l}\text { c. } 2408 \mathrm{G}>\mathrm{A} \text { (p.Arg803His) } \\
\text { Exon } 24\end{array}$ & 59 & $\begin{array}{l}\text { Creation of an exonic ESS site. Potential alteration of splicing. } \\
(1-E S R \text { sequences from Goren et al. }-2 \text { - IIEs from Zhang et al. }-3 \text {-Sironi et al. - Motif 1) }\end{array}$ & No effect on splicing \\
\hline $\begin{array}{l}\text { c. } 2456 \mathrm{G}>\mathrm{A}(\mathrm{p} . \operatorname{Arg} 819 \mathrm{Gln}) \\
\text { Exon } 24\end{array}$ & 59 & $\begin{array}{l}\text { Activation of an exonic cryptic acceptor site, with the presence of one or more cryptic branch } \\
\text { point(s). Potential alteration of splicing. } \\
(1-\text { HSF matrices: } 65.5-94.44-+44.18-2-\text { MaxEnt: } 0.35-8.31-+2474.29) \\
\text { Creation of an exonic ESS site. Potential alteration of splicing. } \\
\text { (1- Sironi et al. }- \text { Motif } 2-2-\text { HSF matrices - hnRNP A1) }\end{array}$ & No effect on splicing \\
\hline $\begin{array}{l}\text { c. } 3826 \mathrm{C}>\mathrm{G}(\mathrm{p} . \mathrm{Leu} 1276 \mathrm{Val}) \\
\text { Exon } 34\end{array}$ & 40 & $\begin{array}{l}\text { Activation of an exonic cryptic donor site. Potential alteration of splicing. } \\
(1-\text { HSF matrices: } 51.89-78.72-+51.71) \\
\text { Creation of an exonic ESS site. Potential alteration of splicing. } \\
(1-\text { Sironi et al. }- \text { Motif } 2-2-\text { HSF matrices }- \text { hnRNP A1) }\end{array}$ & No effect on splicing \\
\hline $\begin{array}{l}\text { c.5201A }>\mathrm{G} \text { (p.Glu1734Gly) } \\
\text { Exon } 47\end{array}$ & 100 & 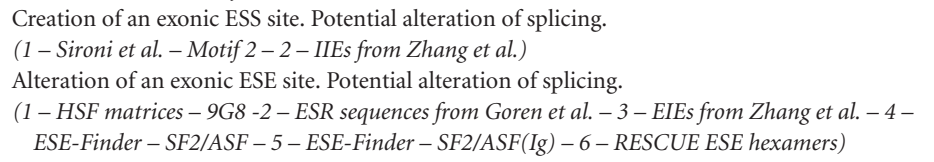 & No effect on splicing \\
\hline $\begin{array}{l}\text { c.797T }>C \text { (p.Leu266Pro) } \\
\text { Exon } 8\end{array}$ & 69 & No significant splicing motif alteration detected & No effect on splicing \\
\hline $\begin{array}{l}\text { c. } 845 \mathrm{~T}>\mathrm{C}(\mathrm{p} . \mathrm{Ile} 282 \mathrm{Thr}) \\
\text { Exon } 8\end{array}$ & 88 & No significant splicing motif alteration detected & No effect on splicing \\
\hline $\begin{array}{l}\text { c. } 965 T>C \text { (p.Leu322Pro) } \\
\text { Exon } 11\end{array}$ & 71 & No significant splicing motif alteration detected & No effect on splicing \\
\hline $\begin{array}{l}\text { c. } 1020 \mathrm{C}>\mathrm{A}(\mathrm{p} . \operatorname{Ser} 340 \mathrm{Arg}) \\
\text { Exon } 11\end{array}$ & 71 & No significant splicing motif alteration detected & No effect on splicing \\
\hline $\begin{array}{l}\text { c. } 1020 \mathrm{C}>\mathrm{G}(\mathrm{p} . \text { Ser340Arg }) \\
\text { Exon } 11\end{array}$ & 71 & No significant splicing motif alteration detected & No effect on splicing \\
\hline $\begin{array}{l}\text { c.6196G }>\text { A (p.Ala2066Thr) } \\
\text { Exon } 54\end{array}$ & 71 & No significant splicing motif alteration detected & No effect on splicing \\
\hline
\end{tabular}

In dysferlinopathies, dysferlin is absent or strongly reduced in patient biopsies. Missense mutations, which represent $33.1 \%$ of mutations in DYSF [Blandin et al., 2012], could perturb splicing. Indeed, modifications of the sequence of the protein could lead to a wrong localization of the protein or perturb interactions with partners; nevertheless, these proteins could be produced and stable.
Therefore, deleterious effects on splicing, which mostly lead to the absence of protein, could explain the absence or reduction of dysferlin. The relative easy technical procedure of the minigene strategy could allow the study of the effect on splicing for these mutations.

We have shown that missense mutations c.565C $>\mathrm{G}$ and c. $1555 \mathrm{G}>\mathrm{A}$ created new $5^{\prime}$ and $3^{\prime}$ splice sites, respectively. For 


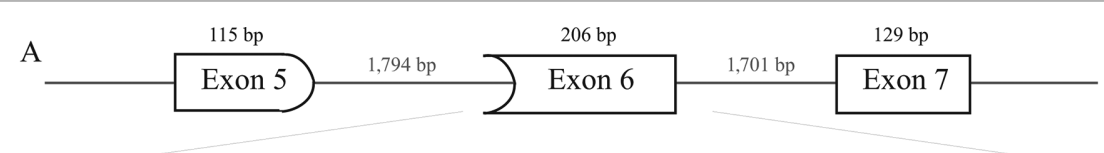

ccaggcctcattagggecctctcctttagACACAGGAGGAGAGGAAGACACAGAGGACCAGGGACTCACTGGAGATGAGGCGGAGCCATT

CCTGGATCAAAGCGGAGGCCCGGGGGCTCCCACCACCCCAAG/GAAA(C)TACCTTCACGTCCTCCGCCCCACTACCCCGGGA TCAAAAGAAAGCGAAGTGCGCCTACATCTAGAAAGCTGCTGTCAGACAAACCGCAGGATTTCCAGgtgatgaacgggetttctetgaccecaggc

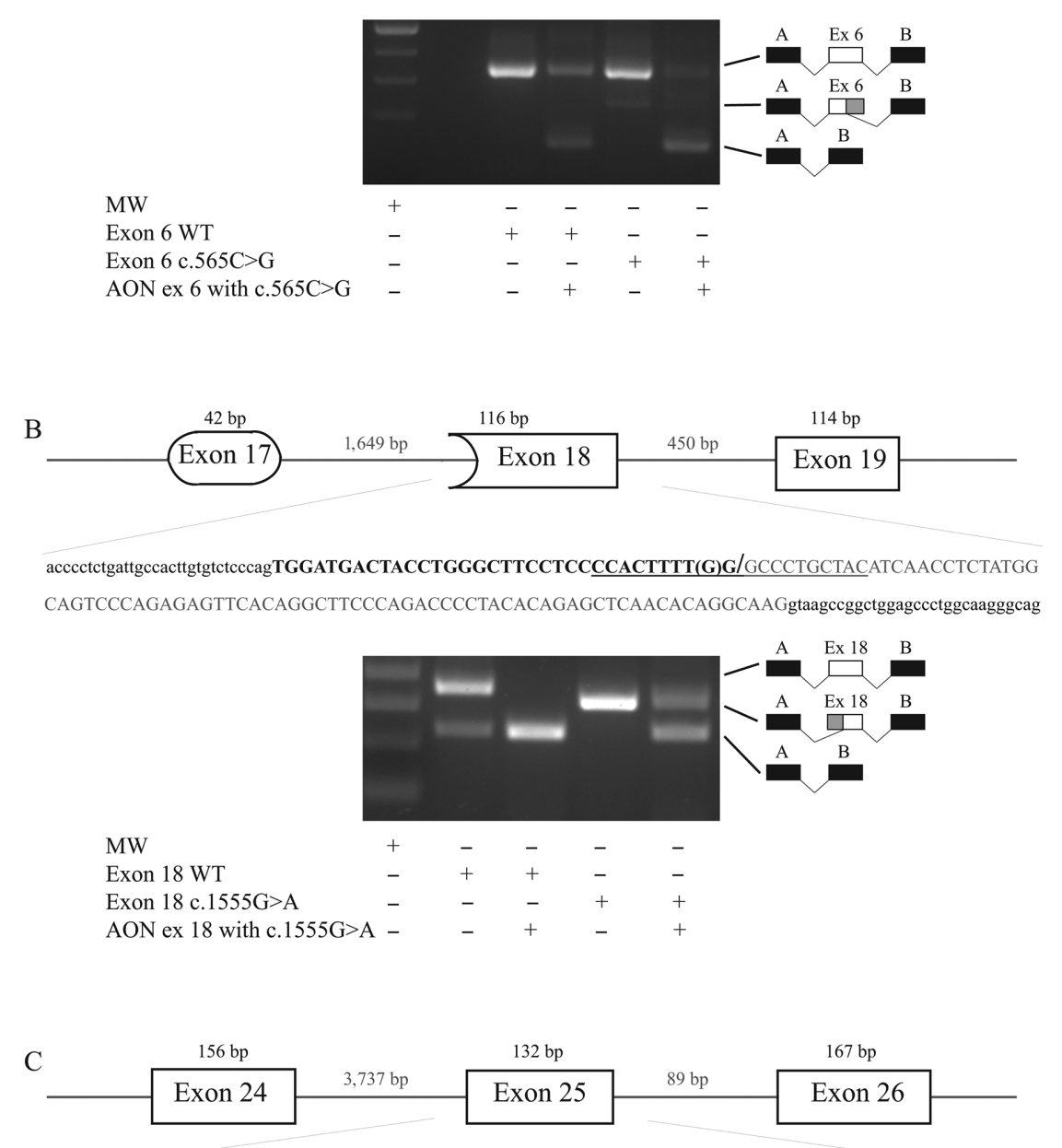

tgtgggtttctgtccttctctggtacccagTATCCGATGGAGAAGGTGCCTGGCGCCCGGATGCCAGTGCAGATACGGGTCAAGCTGTGGTTTGGGC TCTCAGTGGATGAGAAGGAGTTCAACCAGTTTGCTGAGGGGAAGCTGTCTGTCTTTGCTGAA(A)CCgtgagtacctgccagcceccacctctgcct

MW

Exon 25 WT

Exon 25 c. $2641 \mathrm{~A}>\mathrm{C}$

AON ex 25

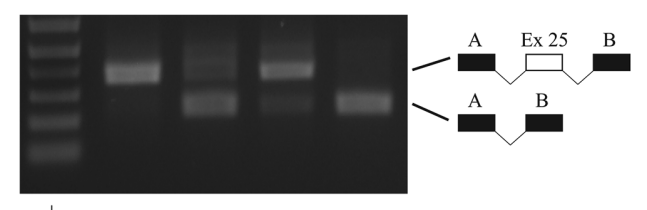

$\begin{array}{ccccc}+ & - & - & - & - \\ - & + & + & - & - \\ - & - & - & + & + \\ - & - & + & - & +\end{array}$

Figure 2. Mutations altering auxiliary splicing signals. We used the splicing reporter minigene assay and AON to detect the presence of auxiliary splicing signals. In each case, transfections into HEK 293 cells were performed both using WT and mutant pCAS2 constructions with and without AON. Forty-eight hours after transfection, transcriptional analysis was performed. The scheme presents the phasing of exons and the size of intron/exon. Intronic sequences are indicated in lowercase letters and exonic sequences are indicated with bold capital letters. Deletions due to the mutation are in gray letters; mutations are in black and parenthesis. Regions targeted by AON are underlined. A: Exon skipping due to the AON targeting the sequence between c.555 and c.574 in exon 6 is observed both in WT and mutant carrying the mutation c.565C $>$ G. Transcript of $339 \mathrm{bp}$ due to the mutation c. $565 \mathrm{C}>\mathrm{G}$ is also seen. B: Exon skipping due to the $\mathrm{AON}$ targeting the sequence between c. 1547 and c. 1566 in exon 18 is observed both in WT and mutant carrying the mutation c. $1555 \mathrm{G}>\mathrm{A}$. Transcript of 318 bp due to the mutation c. $1555 \mathrm{G}>\mathrm{A}$ is also seen. C: Exon skipping due to the AON targeting the locus c.2622-c.2636 in exon 25 is observed both in WT and mutant carrying the mutation c.2641A>C. Transcript of 236 bp due to the mutation c. $2641 \mathrm{~A}>\mathrm{C}$ is also seen. 
mutation $c .565 \mathrm{C}>\mathrm{G}$, two $5^{\prime}$ splice sites are predicted by HSF, whereas only the stronger site (72.21) was used and detected by the minigene assay. Concomitantly, these mutations also abolished an ESE and created an ESS, which prevented the use of the natural splice sites. Surprisingly, the new splice sites created by the mutation c. $565 \mathrm{C}>\mathrm{G}$ and $\mathrm{c} .1555 \mathrm{G}>\mathrm{A}$ have a lower $\mathrm{CV}$ than the natural site, but have been selected by the spliceosome. We hypothesized that this is explained by the concomitant modification of auxiliary splicing signals that result in natural splice site nonrecognition. Furthermore, this phenomenon could explain the differences between the minigene assay and HSF predictions. Indeed, for all predictions related to the creation of a new splice site by missense mutations, for which we showed no effect, the $\mathrm{CV}$ of the site created by the mutations is lower than the natural splice sites, and no ESE is predicted to be disrupted by the mutation.

It has been previously suggested that exons with reduced splice sites' CV might require auxiliary splicing signals to ensure exon inclusion [Pagani et al., 2005; Gaildrat et al., 2012]. We showed that missense mutations c.463G $>\mathrm{A}$ and c.2641A $>\mathrm{C}$ abolished an ESE leading to a partial exon skipping. These mutations are located in exon 6 and 25, respectively, which harbor weak $3^{\prime}$ and $5^{\prime}$ splice sites.

Understanding the specific physiopathology of pathogenic mutations such as splicing defects could help develop allele-specific therapies. Indeed, it has been previously shown that the use of AON directed against mutations, which lead to the creation of pseudoexons or new $5^{\prime}$ or $3^{\prime}$ splice sites, may restore the use of natural splice sites [Hammond and Wood, 2011; Blázquez et al., 2013].

We have shown that some mutations leading to exon skipping (exons 9, 25, 34, and 49) preserve the reading frame. These mutations have been found in patient's genomes, indicating that they are probably pathogenic. Thus, the corresponding deleted regions could be important for dysferlin's functions or the deletion could destabilize the protein, leading to its degradation. Regarding the exon 32 , it has been previously shown that skipping of this in-frame exon produces a functional truncated protein [Sinnreich et al., 2006; Azakir et al., 2012b]. The exon-skipping strategy may therefore be used for this specific exon to rescue nonsense mutations [Wein et al., 2010].

Additionally, our results confirm the efficiency of HSF [Desmet et al., 2009] for the detection of splicing abnormalities. Indeed, HSF was $100 \%$ accurate for the detection of splicing defect due to mutations. However, our minigene strategy should be combined to HSF predictions as predictions for auxiliary sequence modifications may produce false-positive results. Indeed, for $44 \%$ of missense mutations tested (five with deleterious effect and six without deleterious effect), we confirmed the prediction. Nevertheless, we evidenced no deleterious effect for $56 \%$ of the tested mutations ( 14 mutations) for which a deleterious effect was predicted. Noteworthy, the majority of these false predictions (10 out of 14) concerned the auxiliary sequences, and the other four both auxiliary sequences and splice sites activation. Similar limitations are encountered with other bioinformatics tools available for the prediction of splicing defects involving ESE/ESS, warranting the use of functional assays such as the test we applied to further improve predictor tools [Desmet et al., 2009].

Forty-six mutations affecting splicing have been reported among the 266 different mutational events listed in UMD-DYSF. They include both intronic and missense mutations. Intronic mutations account for $14.8 \%$ of all DYSF alleles, whereas missense mutations account for $32.1 \%$ of alleles. We showed here that $20 \%$ of missense mutations tested impact splicing, a proportion consistent with previous estimations made on the dataset of human disease alleles, which indicated that $22 \%$ of disease alleles that were originally clas- sified as missense mutations may also affect splicing [Lim et al., 2011]. If we take into account that $20 \%$ of missense mutations indeed impact splicing (as demonstrated in our study), we can predict that up to $21.2 \%$ of DYSF alleles have an impact on splicing (without considering nonsense mutations or indels that may also result in splicing defects). This could be compared with reported data for the neurofibromin gene for which up to $50 \%$ of mutations alter splicing [Ars et al., 2000]. Thus, the percentage of mutations of the DYSF gene that affect splicing is probably underevaluated.

The characterization of splicing defects is of prime importance not only for a better understanding of natural splicing mechanism, but also for the development and improvement of predictive tools. A better prediction of the effect of mutations for which a deleterious effect is not obvious, such as intronic, missense, and isosemantic mutations, will be indispensable to conclude regarding pathogenicity of mutations identified in the new era of next-generation sequencing approaches.

\section{Acknowledgments}

We thank patients and their relative for their participation in this study. We would like to thank Tangui Messina and Francesca Puppo for technical assistance. We also acknowledge Alexandra Martins and Christiane Duponchel for the gift of the pCAS2 vector.

\section{References}

Aoki M, Liu J, Richard I, Bashir R, Britton S, Keers SM, Oeltjen J, Brown HE, Marchand S, Bourg N, Beley C, McKenna-Yasek D, et al. 2001. Genomic organization of the dysferlin gene and novel mutations in Miyoshi myopathy. Neurology 57:271278.

Ars E, Serra E, García J, Kruyer H, Gaona A, Lázaro C, Estivill X. 2000. Mutations affecting mRNA splicing are the most common molecular defects in patients with neurofibromatosis type 1. Hum Mol Genet 9:237-247.

Azakir BA, Di Fulvio S, Kinter J, Sinnreich M. 2012a. Proteasomal inhibition restores biological function of mis-sense mutated dysferlin in patient-derived muscle cells. J Biol Chem 287:10344-10354.

Azakir BA, Di Fulvio S, Salomon S, Brockhoff M, Therrien C, Sinnreich M. 2012b. Modular dispensability of dysferlin C2 domains reveals rational design for minidysferlin molecules. J Biol Chem 287:27629-27636.

Bansal D, Miyake K, Vogel SS, Groh S, Chen C-C, Williamson R, McNeil PL, Campbell KP. 2003. Defective membrane repair in dysferlin-deficient muscular dystrophy. Nature 423:168-172.

Bashir R, Britton S, Strachan T, Keers S, Vafiadaki E, Lako M, Richard I, Marchand S, Bourg N, Argov Z, Sadeh M, Mahjneh I, et al. 1998. A gene related to Caenorhabditis elegans spermatogenesis factor fer-1 is mutated in limb-girdle muscular dystrophy type 2 B. Nat Genet 20:37-42.

Blandin G, Beroud C, Labelle V, Nguyen K, Wein N, Hamroun D, Williams B, Monnier N, Rufibach LE, Urtizberea JA, Cau P, Bartoli M, et al. 2012. UMD-DYSF, a novel locus specific database for the compilation and interactive analysis of mutations in the dysferlin gene. Hum Mutat 33:E2317-E2331.

Blázquez L, Aiastui A, Goicoechea M, Martins de Araujo M, Avril A, Beley C, García L, Valcárcel J, Fortes P, López de Munain A. 2013. In vitro correction of a pseudoexongenerating deep intronic mutation in LGMD2A by antisense oligonucleotides and modified small nuclear RNAs. Hum Mutat 34:1387-1395.

Cacciottolo M, Numitone G, Aurino S, Caserta IR, Fanin M, Politano L, Minetti C, Ricci E, Piluso G, Angelini C, Nigro V. 2011. Muscular dystrophy with marked Dysferlin deficiency is consistently caused by primary dysferlin gene mutations. Eur J Hum Genet 19:974-980.

Cagliani R, Magri F, Toscano A, Merlini L, Fortunato F, Lamperti C, Rodolico C, Prelle A, Sironi M, Aguennouz M, Ciscato P, Uncini A, et al. 2005. Mutation finding in patients with dysferlin deficiency and role of the dysferlin interacting proteins annexin A1 and A2 in muscular dystrophies. Hum Mutat 26:283.

Cartegni L, Chew SL, Krainer AR. 2002. Listening to silence and understanding nonsense: exonic mutations that affect splicing. Nat Rev Genet 3:285-298.

Desmet F-O, Hamroun D, Lalande M, Collod-Béroud G, Claustres M, Béroud C. 2009. Human Splicing Finder: an online bioinformatics tool to predict splicing signals. Nucleic Acids Res 37:e67.

Frédéric MY, Lalande M, Boileau C, Hamroun D, Claustres M, Béroud C, CollodBéroud G. 2009. UMD-predictor, a new prediction tool for nucleotide substitution 
pathogenicity-application to four genes: FBN1, FBN2, TGFBR1, and TGFBR2. Hum Mutat 30:952-959.

Fujita E, Kouroku Y, Isoai A, Kumagai H, Misutani A, Matsuda C, Hayashi YK, Momoi T. 2007. Two endoplasmic reticulum-associated degradation (ERAD) systems for the novel variant of the mutant dysferlin: ubiquitin/proteasome $\operatorname{ERAD}(\mathrm{I})$ and autophagy/lysosome ERAD(II). Hum Mol Genet 16:618-629.

Gaildrat P, Killian A, Martins A, Tournier I, Frébourg T, Tosi M. 2010. Use of splicing reporter minigene assay to evaluate the effect on splicing of unclassified genetic variants. Methods Mol Biol 653:249-257.

Gaildrat P, Krieger S, Di Giacomo D, Abdat J, Révillion F, Caputo S, Vaur D, Jamard E, Bohers E, Ledemeney D, Peyrat J-P, Houdayer C, et al. 2012. Multiple sequence variants of BRCA2 exon 7 alter splicing regulation. J Med Genet 49:609-617.

Hammond SM, Wood MJA. 2011. Genetic therapies for RNA mis-splicing diseases. Trends Genet 27:196-205.

Lim KH, Ferraris L, Filloux ME, Raphael BJ, Fairbrother WG. 2011. Using positional distribution to identify splicing elements and predict pre-mRNA processing defects in human genes. Proc Natl Acad Sci USA 108:11093-11098.

Liu J, Aoki M, Illa I, Wu C, Fardeau M, Angelini C, Serrano C, Urtizberea JA, Hentati F, Hamida MB, Bohlega S, Culper EJ, et al. 1998. Dysferlin, a novel skeletal muscle gene, is mutated in Miyoshi myopathy and limb girdle muscular dystrophy. Nat Genet 20:31-36.

DeLuna N, Freixas A, Gallano P, Caselles L, Rojas-García R, Paradas C, Nogales G, Dominguez-Perles R, Gonzalez-Quereda L, Vílchez JJ, Márquez C, Bautista J, et al. 2007. Dysferlin expression in monocytes: a source of mRNA for mutation analysis. Neuromuscul Disord 17:69-76.
Pagani F, Raponi M, Baralle FE. 2005. Synonymous mutations in CFTR exon 12 affect splicing and are not neutral in evolution. Proc Natl Acad Sci USA 102:63686372.

Sinnreich M, Therrien C, Karpati G. 2006. Lariat branch point mutation in the dysferlin gene with mild limb-girdle muscular dystrophy. Neurology 66:11141116.

Smith CW, Chu TT, Nadal-Ginard B. 1993. Scanning and competition between AGs are involved in 3' splice site selection in mammalian introns. Mol Cell Biol 13:4939_ 4952.

Smith CW, Porro EB, Patton JG, Nadal-Ginard B. 1989. Scanning from an independently specified branch point defines the 3' splice site of mammalian introns. Nature 342:243-247.

Therrien C, Dodig D, Karpati G, Sinnreich M. 2006. Mutation impact on dysferlin inferred from database analysis and computer-based structural predictions. J Neurol Sci 250:71-78.

Thusberg J, Olatubosun A, Vihinen M. 2011. Performance of mutation pathogenicity prediction methods on missense variants. Hum Mutat 32:358-368.

Wein N, Avril A, Bartoli M, Beley C, Chaouch S, Laforêt P, Behin A, Butler-Browne G, Mouly V, Krahn M, Garcia L, Lévy N. 2010. Efficient bypass of mutations in dysferlin deficient patient cells by antisense-induced exon skipping. Hum Mutat 31:136-142.

Wenzel K, Carl M, Perrot A, Zabojszcza J, Assadi M, Ebeling M, Geier C, Robinson PN, Kress W, Osterziel K-J, Spuler S. 2006. Novel sequence variants in dysferlindeficient muscular dystrophy leading to mRNA decay and possible C2-domain misfolding. Hum Mutat 27:599-600. 